\title{
Opinião de mães e professoras sobre o uso de um protocolo de observação do desempenho de crianças com deficiência física
}

\section{Mothers' and teachers' opinions about the use of an observation protocol of performance of children with physical disabilities}

\author{
Mariana Dutra Zafani ${ }^{1}$, Sadao Omote ${ }^{2}$, Luciana Ramos Baleotti ${ }^{3}$
}

http://dx.doi.org/10.11606/issn.2238-6149.v27i1p36-41

\begin{abstract}
Zafani MD, Omote S, Baleotti LR. Opinião de mães e professoras sobre o uso de um protocolo de observação do desempenho de crianças com deficiência física. Rev Ter Ocup Univ São Paulo. 2016 jan.-abr.;27(1):36-41.

RESUMO: Objetivou-se descrever e analisar a opinião de mães e de professoras sobre o uso do Protocolo de Observação do Desempenho de Crianças com Deficiência Física na Realização de Atividades de Pintura e Colagem. Participaram 13 mães e 13 professoras de crianças com deficiência física. As participantes tinham duas tarefas. Primeiro, preencher o Protocolo com base na observação de uma filmagem da criança desempenhando uma atividade de pintura e colagem. $\mathrm{Na}$ sequência, responder a um roteiro de entrevista que tinha como objetivo identificar as suas opiniões acerca do uso deste Protocolo. Os dados foram analisados qualitativamente. Os resultados sugerem que o Protocolo é de fácil preenchimento e de linguagem acessível para ambos os grupos. $\mathrm{O}$ seu uso representou uma possibilidade para identificar informações referentes ao desempenho da criança que, em alguma medida, podem contribuir para a atuação do professor e dos pais frente à realização de atividades escolares.
\end{abstract}

DESCRITORES: Desempenho psicomotor; Pessoas com deficiência; Mães; Docentes; Crianças com deficiência/educação.
Zafani MD, Omote S, Baleotti LR. Mothers' and teachers' opinions about the use of an observation protocol of performance of children with physical disabilities. Rev Ter Ocup Univ São Paulo. 2016 Jan.-Apr.;27(1):36-41.

ABSTRACT: This paper aims to describe and analyze mothers' and teachers' opinions about use of an Observation Protocol of The Performance of Children With Physical Disabilities During Painting and Aollage Cctivities. A total of 13 mothers and 13 teachers of children with physical disabilities participated in the research. The participants had two tasks. First, fill in the Protocol after watching a recorded painting and collage activity. Subsequently, answer to an interview script prepared to identify their opinions regarding the use of this Protocol. The collected data were analyzed qualitatively. The results suggest that the Protocol is easy to fill in and the language is accessible to both groups. Its use enabled the identification of information related to the child's performance that, to some extent, may help teachers and parents carrying out school activities.

KEYWORDS: Psychomotor performance; People with physical disabilities; Mothers; Teachers; Children with physical disabilities/education.

O artigo relata parte do estudo apresentado na Dissertação de Mestrado defendida pela primeira autora, disponível na biblioteca da Faculdade de Filosofia e Ciências, Unesp, campus de Marília. - Apoio financeiro: CAPES. Resultados parciais deste estudo foram apresentados: V Congresso Brasileiro de Educação Especial, VII Encontro Nacional dos Pesquisadores da Educação Especial, São Carlos, $14-17$ nov. 2012; VII Congresso Brasileiro Multidisciplinar de Educação Especial, VIII Encontro da Associação Brasileira de Pesquisadores em Educação Especial, Londrina, PR, 5-07 nov. 2013.

1. Faculdade de Filosofia e Ciências, UNESP, campus de Marília, SP.

2. Faculdade de Filosofia e Ciências, UNESP, campus de Marília, SP. E-mail: somote@uol.com.br

3. Faculdade de Filosofia e Ciências, UNESP, campus de Marília, SP. E-mail: baleotti@marília.unesp.br

Endereço para correspondência: Av. Hygino Muzzi Filho, 737, Caixa Postal 181, Bairro: Campus Universitário. Marília, SP. E-mail: marianadz@ig.com.br 


\section{INTRODUÇÃO}

$\mathrm{O}$ "Protocolo de Observação do Desempenho de Crianças com Deficiência Física na Realização de Atividades de Pintura e Colagem" foi desenvolvido para mensurar a percepção dos pais e a dos professores acerca de dois aspectos do desempenho de crianças com deficiência física em uma atividade habitualmente realizada no contexto escolar. Esses aspectos referem-se à capacidade ou incapacidade e independência ou dependência com que as crianças desempenham as etapas envolvidas numa atividade de pintura e colagem ${ }^{1}$.

O interesse por estudar as percepções dos pais e dos professores acerca do desempenho da criança reside na importância de se ponderar a influência de suas variáveis internas (valores, crenças, expectativas, preconceito, estereótipos, etc) e do meio social no qual cada um deles está inserido sobre a formação do percepto em relação à criança com deficiência ${ }^{2}$. O percebedor acaba por informar sobre a sua perspectiva e projeta, muitas vezes, seus sentimentos e crenças em relação à pessoa observada. Esse processo pode determinar a natureza da interação entre as pessoas, implicando, entre outras consequências, maior ou menor acolhimento, expectativas e exigências.

"Estudos da natureza da percepção de uma mesma criança, na perspectiva dos familiares e na dos professores, podem contribuir para a compreensão do envolvimento desses atores no processo inclusivo dessa criança em contextos escolares" (p.24) ${ }^{3}$.

É preciso criar condições ou procedimentos para que os pais e professores se sintam capazes de contribuir para o processo de desenvolvimento da criança e participar ativamente desse processo. Nesse sentido, a oportunidade da observação do desempenho da criança em uma mesma atividade por pais e professores, pode direcionar a atenção para detalhes específicos do desempenho e, consequentemente, favorecer a expressão da opinião sobre as possibilidades e dificuldades da criança. Uma vez que os pais e professores estão observando com cuidado os mesmos aspectos do desempenho, há elementos concretos e específicos que podem contribuir para o diálogo sobre impressões e percepções.

A família e a escola são consideradas como grandes responsáveis pela promoção do desenvolvimento humano atuando como propulsoras ou inibidoras do crescimento intelectual, físico, emocional e social do indivíduo ${ }^{4}$.
$\mathrm{Na}$ escola, "o atendimento às necessidades cognitivas, psicológicas, sociais e culturais da criança é realizado de uma maneira mais estruturada e pedagógica que no ambiente de casa" (p.304)5. Já no âmbito familiar, os métodos, objetivos e conteúdos se distinguem, fomentando o processo de socialização, a proteção e $o$ desenvolvimento de seus membros no plano afetivo, social e cognitivo ${ }^{4}$.

Marcondes e Sigolo ${ }^{6}$ destacaram a necessidade de haver colaboração e comunicação entre pais e professores, a fim de que os contextos familiar e escolar tornem-se benéficos ao desenvolvimento integral da criança. No entanto, essa relação nem sempre ocorre de maneira harmoniosa e satisfatória, pois muitas vezes as expectativas de cada parte envolvida não são atendidas ${ }^{7}$. Os pais e os professores precisam conhecer o que cada um espera da criança, acredita ou percebe que ela é capaz de fazer, pois é através da troca de informações entre eles que será possível visualizarem as potencialidades e limitações da criança e discutirem sobre elas ${ }^{1}$.

Embora o Protocolo de Observação do Desempenho de Crianças com Deficiência Física ainda não tenha sido utilizado com a proposição de investigar a sua contribuição para a aproximação entre família e escola, supõe-se que a forma como deve ser utilizado e pontuado pode possibilitar a aproximação.

Por meio da produção de filmagens de crianças com deficiência física na execução de atividades de pintura e colagem, pais e professores assinalam, separadamente, a alternativa de resposta que se enquadra ao seu julgamento em relação ao desempenho da criança. Esse procedimento permitiu, em estudo anterior ${ }^{1}$, identificar a percepção de cada um desses sujeitos acerca do desempenho da criança, as divergências e as convergências nas percepções. Ao se pesquisar sobre as semelhanças e diferenças na percepção de pais e professores procura-se esclarecer questões que podem contribuir para a construção de uma relação consonante entre eles ${ }^{1}$. A construção dessa relação pode ser investigada em estudos futuros por meio do uso do Protocolo de Observação do Desempenho de Crianças com Deficiência Física na Realização de Atividades de Pintura e Colagem. Para tanto, esse Protocolo necessita possuir uma linguagem acessível e de fácil compreensão para seus usuários.

Nessa perspectiva, o objetivo geral deste estudo é o de descrever e analisar a opinião de mães e de professoras acerca do uso deste Protocolo de Observação. Os objetivos específicos são: identificar aspectos relativos à facilidade e/ou dificuldade no uso, a adequação semântica dos itens e a opinião quanto à experiência em observar o desempenho da criança. 


\section{PROCEDIMENTOS METODOLÓGICOS}

O presente estudo foi aprovado pelo Comitê de Ética em Pesquisa da Faculdade de Filosofia e Ciências da Universidade Estadual Paulista - FFC/UNESP/Marília SP sob o protocolo de número 0125/2011.

Participaram deste estudo treze professoras que atuavam com alunos com deficiência física, na idade de 4 a 9 anos, sendo três professoras da Educação Infantil, nove do Ensino Fundamental e uma da Educação Especial. A idade das professoras variou de 29 a 53 anos. Das treze professoras, doze tinham formação em Pedagogia e uma em magistério.

Além destas, foram incluídas no estudo as treze mães dessas crianças com deficiência física. A idade das mães variou de 25 a 42 anos. No que se refere à escolaridade, uma mãe possuia o Ensino Fundamental completo, duas o Ensino Fundamental incompleto, quatro o Ensino Médio, outras quatro o Ensino Médio incompleto e duas mães o Ensino Superior completo. Todas as participantes assinaram o Termo de Consentimento Livre e Esclarecido.

Para a coleta de dados, foram utilizados dois instrumentos, descritos abaixo:

1 - Protocolo de Observação do Desempenho de Crianças com Deficiência Física na Realização de Atividades de Pintura e Colagem, desenvolvido por Zafani ${ }^{1}$ e tem como foco identificar a percepção dos pais e professores acerca do desempenho de crianças com deficiência física, na faixa etária entre 4 e 9 anos. As atividades desenvolvidas pelas crianças foram escolhidas em função da sua adequação para o propósito de observação detalhada por parte dos pais e professores. A padronização da atividade foi uma exigência metodológica. Vale frisar que não houve pretensão alguma de educar as crianças por meio da atividade elaborada, uma vez que o foco do Protocolo não recai sobre a aprendizagem do aluno com deficiência nem na sua inclusão. Este Protocolo ao pesquisar sobre as semelhanças e diferenças na percepção entre família e escola, sinaliza para a importância de, no contexto da educação inclusiva, haver consonância entre esses contextos ${ }^{1}$.

As atividades foram previamente selecionadas e analisadas com o intuito de adequá-las às competências motoras, cognitivas e perceptivas das crianças de diferentes idades. Foram elaboradas três atividades de pintura e colagem com graus de dificuldades diferenciados tais como quantidade de peças a ser colada, complexidade dos desenhos, habilidade para discriminar figura-fundo e complexidade da colagem ${ }^{1}$.

Este Protocolo constitui-se de 20 itens, distribuídos em duas seções. A seção 1 contém 13 itens e a seção 2 contém 7. A seção 1 , de habilidades motoras, pontua a eficiência da criança ao movimentar os objetos e a si própria no ambiente. A seção 2, de habilidades de processo, pontua a qualidade do desempenho nas áreas de atenção, compreensão, noção espacial e percepção visual.

Em cada item do Protocolo de Observação, há duas colunas com quatro alternativas de respostas. A Coluna 1 contém quatro alternativas de respostas referentes a capacidade ou incapacidade da criança na execução da atividade: capaz sem dificuldade, capaz com pouca dificuldade, capaz com muita dificuldade e incapaz. A Coluna 2 contém quatro alternativas de respostas referentes a independência ou dependência da criança na execução da atividade: não precisa de ajuda, precisa de pouca ajuda, precisa de muita ajuda $\mathrm{e}$ a terapeuta executa este passo da tarefa sem a participação da criança.

Os participantes assistem a filmagem do seu (sua) filho (a) ou aluno (a), realizando uma atividade de pintura e colagem e, com base na observação dessa filmagem, preenchem o Protocolo. As mães e as professoras assinalam uma alternativa de resposta da coluna 1 e uma alternativa de resposta da coluna 2, em todos os itens que compõem o Protocolo.

Para mensurar a percepção dos pais e dos professores a partir do Protocolo preenchido por eles, é necessário que observem uma mesma situação padronizada de desempenho da criança com deficiência física, em virtude disso, convencionou-se o uso da filmagem.

2 - Roteiro de entrevista estruturado, composto por três questões. Uma questão é fechada com cinco alternativas de resposta: muito fácil de preencher, fácil de preencher, nem fácil e nem difícil, difícil de preencher e muito difícil de preencher. No caso de o respondente assinalar uma das duas últimas alternativas, deve justificar sua escolha.

Duas questões são abertas e têm a finalidade de identificar a opinião das participantes quanto à existência de itens de difícil compreensão e o motivo de tal dificuldade, bem como a opinião quanto à experiência de observar o desempenho da criança por meio da filmagem.

Foram agendados com as mães e as professoras, individualmente, o dia e horário em que procederiam à observação da filmagem, o preenchimento do Protocolo e a resposta ao roteiro de entrevista estruturado. As professoras desenvolveram essa atividade na própria escola em que seus alunos estavam inseridos e as mães na clínica de reabilitação em que as crianças recebiam atendimento.

As verbalizações foram gravadas e transcritas na íntegra, para posterior análise das falas. Os relatos foram lidos e examinados detalhadamente para identificar e 
organizar as unidades de análise, as quais se constituíram por trechos de falas agrupados por critério de semelhança temática. Nessa análise foram delineados três eixos:

- Grau de facilidade ou dificuldade no preenchimento do Protocolo que contempla a análise das participantes em relação ao grau de facilidade ou dificuldade no uso do Protocolo;

- Compreensão quanto ao enunciado dos itens que verifica se houve dificuldade para a compreensão do conteúdo de cada um dos itens e o motivo para tal dificuldade;

- Opinião relativa à experiência em observar o desempenho da criança que ilustra por relatos que possuem em seu conteúdo a atribuição de adjetivos relativos à experiência vivenciada; e verbalizações que ilustram a autoavaliação das participantes em relação as suas ações frente à criança e a opinião delas quanto às habilidades da criança.

\section{RESULTADOS E DISCUSSÃO}

Os resultados estão organizados por meio de eixos de análise. As professoras são identificadas pela letra $\mathrm{P}$ e as mães pela letra $\mathrm{M}$.

\section{Eixo 1 - Grau de facilidade ou dificuldade no preenchimento do Protocolo}

Apresenta-se, neste eixo, a análise da opinião das participantes quanto à facilidade ou dificuldade no uso do Protocolo. A Tabela 1 mostra os resultados encontrados.

Tabela 1 - Grau de facilidade ou dificuldade no preenchimento do Protocolo

\begin{tabular}{l|c|c|c|c|c}
\hline \multirow{2}{*}{ Participantes } & \multicolumn{5}{|c}{ Alternativas assinaladas } \\
\cline { 2 - 6 } & $\begin{array}{c}\text { Muito } \\
\text { fácil }\end{array}$ & Fácil & $\begin{array}{c}\text { Nem fácil e } \\
\text { nem difícil }\end{array}$ & Difícil & $\begin{array}{c}\text { Muito } \\
\text { difícil }\end{array}$ \\
\hline Professoras & 3 & 9 & 1 & 0 & 0 \\
\hline Mães & 4 & 9 & 0 & 0 & 0 \\
\hline
\end{tabular}

Fonte: Elaborada pelos autores.

Os dados apontam que para a maioria das participantes o Protocolo apresenta facilidade em seu uso.

Destaca-se que M3 ao responder esta questão na entrevista mencionou ter apresentado dificuldades de outras ordens e não de preenchimento do Protocolo em si, conforme indica a fala abaixo:
Senti dificuldade em ver ele fazendo assim, separado, segmentando a atividade. E é difícil também falar e achar que ele é incapaz. (M3).

Constatam-se na verbalização de M3 dois elementos importantes para reflexão. O primeiro refere-se a uma dificuldade em observar o desempenho da criança com base na análise de segmentos que compõem a atividade. A título de exemplificação, para responder aos itens do Protocolo tendo como referência o ato de colar, este não era observado de forma global, mas sim frente a todas as etapas que o envolvem, desde pegar, rosquear, apertar, entre outros, esses passos exemplificam o que M3 referiu como "segmentando a atividade".

De fato, para uma mãe esse olhar pode ser complexo, pois representa um olhar voltado para elementos envolvidos antes e durante a execução de uma atividade até chegar a sua finalização. No dia-a-dia, as mães possivelmente observam a realização de atividades feitas pela criança com base em um olhar mais global para o desempenho dela, e não a partir de um olhar fragmentado. Este olhar está presente no conhecimento da Terapia Ocupacional como forma de análise de atividade na perspectiva das demandas da tarefa, da pessoa e do ambiente. Destaca-se que o Protocolo, utilizado neste estudo, foi construído por terapeuta ocupacional, assim, considerou-se importante a observação do desempenho nessa perspectiva.

O segundo aspecto presente na fala de M3 parece ter relação com sentimentos de negação frente à deficiência, amplamente descrito na literatura ${ }^{8,9,10}$. Tal sentimento, talvez tenha emergido no momento em que essa mãe percebeu e referiu a incapacidade que seu filho manifestou no desempenho da atividade observada. De fato, para muitas mães de crianças com deficiência pode ser difícil e até doloroso reconhecer e expor que seu filho apresenta alguma limitação ou é incapaz de realizar uma determinada tarefa. Essa dificuldade pode ter relação com o sentimento de negação frente à condição de deficiência e/ou de incapacidade ${ }^{8}$. Camargo e Londero ${ }^{11}$ referem que, para muitos pais de crianças com deficiência, a negação surge como um dos mecanismos adotado na tentativa de encontrar uma resposta contrária à realidade que se apresenta. Esse mecanismo pareceu muito presente na fala de M3.

\section{Eixo 2 - Compreensão quanto ao enunciado dos itens}

Este eixo foi introduzido para analisar eventual dificuldade das participantes em compreender o conteúdo expresso em cada um dos itens do Protocolo de Observação, bem como averiguar o motivo da possível dificuldade. 
Os resultados indicam que nenhuma das participantes apresentou dificuldade na compreensão, o que sugere que o conteúdo dos itens que compõem o Protocolo, utilizado neste estudo, apresenta-se de forma clara, simples e elucida o que deve ser observado, sem confundir o respondente.

\section{Eixo 3 - Opinião relativa à experiência em observar o desempenho da criança}

De maneira geral, as participantes apresentaram em suas falas adjetivos positivos acerca da experiência da atividade proposta a elas, tais como importante, muito bom, interessante, produtiva, experiência inovadoral diferente, entre outros. Os recortes abaixo exemplificam esses dados.

Achei bom, porque traz um pouco mais de informação [...]. (P2).

Achei uma oportunidade produtiva. (P3).

Achei bastante interessante e fiquei surpresa com o desempenho [...] (M3).

Achei que foi bom deu pra ver as dificuldades dela. (M10).

As professoras enfatizaram que a observação propiciou-lhes a oportunidade de um olhar mais minucioso para o desempenho da criança, o que provavelmente contribuirá para a revisão de sua atuação pedagógica, conforme os relatos abaixo.

Eu achei importante no sentido de ver o que ele consegue fazer. Do jeito que você organizou deu para ver claramente quais são as capacidades que ele tem e as que ele não tem. E faz com que eu possa a partir do que você fez desenvolver até outras atividades que ele é capaz de fazer. (P7).

Eu achei interessante, porque dá para ver como que é a independência dele. Porque aqui ele é muito manhoso, ele pede bastante ajuda na escola e ai na filmagem ele não pediu nenhuma. Foi bom para ver as capacidades. Eu sei que ele faz só que eu tenho que ficar falando para fazer sozinho. Ajudou na minha atuação com ele. (P8).

$E u$ achei muito interessante, porque você no dia-adia não tem condições de estar observando tantos aspectos, como numa filmagem que você pode avaliar mais pontualmente. É diferente, quando você está na sala de aula, você observa as coisas, não tem como não observar, mas você parar para ver por meio da filmagem é diferente. (P13).

Os relatos sugerem que a possibilidade de observar o aluno por meio da filmagem, pode ser uma estratégia útil na formação continuada de professores, e além disso, impactar positivamente na prática pedagógica em sala de aula. Estudos apontam que a observação, análise e reflexão crítica conjunta tem se mostrado importante instrumento para a promoção de mudanças nos relatos e na prática pedagógica do professor, sob a mediação do pesquisador ${ }^{12}$.

Quanto às mães, os relatos sugerem que estas perceberam que subestimavam a capacidade de seus filhos, conforme recortes transcritos abaixo:

Muito boa, porque eu achei que ele foi bem melhor do que eu esperava. Em casa a gente acaba fazendo por ele, não espera ele fazer, que nem você fez. Então foi muito bom. (M2).

Gostei, porque eu nem sabia que ele era capaz de colar, porque eu nunca deixei ele colar, recortar, nunca. Para mim ele não sabia. (M13).

Por meio da filmagem, as mães foram capazes de perceber a capacidade funcional de seu filho, antes não percebida. Tal oportunidade poderia ser ainda mais enriquecida se a observação fosse realizada juntamente com profissionais da saúde e/ou da educação que acompanham a criança ${ }^{1}$. Reflexões que busquem destacar as capacidades que o filho com deficiência apresenta, em vez de orientações sobre o cuidar, ou a necessidade de tarefas a serem estimuladas pelo cuidador, podem influenciar no desencadeamento de sentimentos motivadores de entusiasmo e de expectativas positivas em relação ao desenvolvimento e inserção social do filho com deficiência.

\section{CONSIDERAÇÕES FINAIS}

O objetivo deste relato é apresentar opiniões de mãe e professora sobre o uso do Protocolo de Observação do Desempenho de Crianças com Deficiência Física na Realização de Atividades de Pintura e Colagem. De forma geral, os resultados sugerem que o Protocolo é de fácil compreensão para os seus usuários.

Além disso, os resultados apontam indícios de que este Protocolo pode colaborar para a interface entre 
profissionais da saúde e da educação no sentido de possibilitar a aproximação e reflexão conjunta entre esses sujeitos, na perspectiva da educação inclusiva.

A autoreflexão apresentada pelos professores relativa à oportunidade de revisarem a prática pedagógica, mediante a observação e pontuação sistemática do desempenho da criança, sugere o investimento em pesquisas futuras que busquem levantar dados acerca da possibilidade de profissionais da saúde, sobretudo terapeutas ocupacionais que atuam na educação, utilizar este Protocolo como mediador da formação continuada de professores que atuam com crianças com deficiência física.

\section{REFERÊNCIAS}

1. Zafani MD. Percepção de pais e professores acerca do desempenho de crianças com deficiência física em atividades do contexto escolar [dissertação]. Marília: Universidade Estadual Paulista; 2013. Disponível em: http://base. repositorio.unesp.br/bitstream/handle/11449/91205/zafani md_me_mar.pdf? sequence $=1 \&$ isAllowed $=y$.

2. Zafani MD, Omote S. Protocolo de observação do desempenho de crianças com deficiência física na realização de atividades de pintura e colagem: percepções das mães acerca do uso do instrumento. In: VII Congresso Brasileiro Multidisciplinar de Educação Especial, VIII Encontro da Associação Brasileira de Pesquisadores em Educação Especial. Anais... Londrina, 2013. Londrina: Editora da UEL; 2013. p.2266-75.

3. Zafani MD, Omote S, Baleotti LR. Protocolo de observação do desempenho de crianças com deficiência física: construção, aplicação e análise de dados. Rev Bras Educ Espec. 2015;21(1):23-38. doi: http://dx.doi.org/10.1590/ S1413-65382115000100003.

4. Dessen MA, Polonia AC. A Família e a Escola como contextos de desenvolvimento humano. Paidéia. 2007;17(36):21-32. Disponível em: http://www.scielo.br/ pdf/paideia/v17n36/v17n36a03.pdf.

5. Polonia AC, Dessen MA. Em busca de uma compreensão das relações entre família e escola. Psicol Esc Educ. 2005;9(2):303-12. doi: http://dx.doi.org/10.1590/S141385572005000200012 .

Artigo recebido em: 14.07.15

Artigo aceito em: 23.03.16 